\title{
Dynamic Response of Railway track using two parameter model
}

\author{
Sunita Kumari \\ Deptt. Of Civil Engg. \\ IIT Roorkee-247667 \\ India
}

\author{
Pragyan P Sahoo \\ Deptt. Of Civil Engg. \\ IIT Roorkee-247667 \\ India
}

\author{
V A Sawant \\ Deptt. Of Civil Engg. \\ IIT Roorkee-247667 \\ India
}

\begin{abstract}
In the present analysis, an infinite Euler-Bernoulli beam of constant cross-section resting on an elastic foundation is considered. The beam and foundation are assumed to be homogeneous and isotropic. The foundation is modeled using two parameters with damping. The beam is subjected to a constant point load moving with a constant speed along the beam. An effort has been made to find the solution of the governing differential equation analytically. It gives beam deflection under moving load in closed form for damped case. At subcritical speed the absolute value of the deflection of the beam increases with increase of the load velocity. Peak maximum deflection appears when the load travels at the critical speed.
\end{abstract}

Keywords: CVR, Euler-Bernoulli beam, Winkler beam

\section{INTRODUCTION}

Recent findings indicate one of major causes of damages, which are attributed to the resonant behaviors, in a railway track. Basically, when a railway track is excited to generalize dynamic loading, the railway track deforms and then vibrates for certain duration. Dynamic responses of the railway track and its components are the key to evaluate the structural capacity of railway track and its components. The track vibrations can cause the crack damage in railway sleepers or fasteners, or even the breakage of ballast support. When the foundation is modeled as an elastic foundation, a critical velocity is found for the existing for moving load. Waves excited by a moving load with supercritical velocity propagate in a different way as they do when the load velocity is subcritical. Because high speed vehicles (e.g., trains and automobiles) are getting extensively adopted as the surface transportation carriers, hence considerable attention should be paid to the response of the transportation structure. The main objective of this study is to discuss the vibration and stability of a Bernoulli-Euler beam resting on a Winkler-type elastic foundation subjected to a static axial force under a moving load. A distributed load with a constant advance velocity was considered instead of a point load because moving loads in practice have normally a finite area over which they are distributed and the point load represents only an extreme case. Damping of linear hysteretic nature was considered in this study, it is assumed that the foundation displacement at a point is dependent only on the force acting on the point by conducting parametric studies.

\section{LITERATURE REVIEW}

The approach of beams on elastic foundation is used by many researchers for the analysis of pavement. The problem concerned with the dynamic behavior of an Euler-Bernoulli beam. Excited by a moving load has received considerable attention in civil engineering in the recent years. A detailed literature review based on the assumption of pavement as a beam is outlined here.

Kenny (1954) investigated the effect of viscous damping based on the analytical solution for steady state response of an infinite Euler-Bernoulli beam resting on Winkler foundation. Harr et al. (1969) developed computer program for analyzing beams on elastic foundations, represented by a single layer model whose properties were characterized by two generalized elastic parameters, based on Vlasov's general variational method. Fryba (1972) analyzed the response of an unbounded elastic body subjected to a moving load by using triple Fourier integral transformation. A detailed solution for the problem of a constant moving load along an infinite beam resting on an elastic foundation was presented by considering all possible speeds and values of viscous damping based on the concept of equivalent stiffness of the supporting structures. Two kinds of finite elements were formulated by Zhaohua and Cook (1983) to analyze beams on one or twoparameter foundations based on exact displacement function. Numerical results showed that the element based on the exact displacement function gives exact response even for smaller number of elements. Cifuentes (1989) presented a combined finite element-finite difference technique based on Lagrange Multiplier formulation to study the dynamic response of an Euler-Bernoulli beam excited by a moving mass. Mallik et al. (2006) investigated the steady-state response of EulerBernoulli beam placed on an elastic foundation and subjected to a concentrated load moving with a constant speed. The governing equations in the form of deflection, bending moment and shear force were obtained in closed form for the undamped case. Kien (2008) adopted the FEM to investigate the dynamic response of prestressed Timoshenko beam resting on two-parameter elastic foundation subjected to a moving concentrated harmonic load. It was reported that the critical velocity at which the dynamic deflection of the beam reaches a peak value, is governed by the foundation stiffness. Winkler foundation subject to a platoon of moving loads with uniform line distributions was presented by Sun and Luo (2008). Numerical methods based on the fast Fourier transform were presented for efficient computation of dynamic response of the beam. 


\section{MODELING OF BEAMS ON ELASTIC FOUNDATION SUBJECTED TO MOVING LOADS}

The differential equation of motion for an Euler-Bernoulli beam, resting on a two-parameter foundation and subjected to a moving load is given by

$E I \frac{\partial^{4} w}{\partial x^{4}}-k_{1} \frac{\partial^{2} w}{d x^{2}}+k w+\rho \frac{\partial^{2} w}{\partial t^{2}}+c \frac{d w}{d t}=P \quad x, t \quad E q .1$

Where $\mathrm{w}(\mathrm{x}, \mathrm{t})$ is the transverse deflection of the beam $(\mathrm{m}), \mathrm{E}$ is the Young's modulus of the beam material $(\mathrm{N} / \mathrm{m} 2)$, I is the second moment area of the beam cross section about its neutral axis $(\mathrm{m} 4), \mathrm{k}$ is the spring constant (first parameter)per unit beam length( $\mathrm{N} / \mathrm{m} 2), \mathrm{k} 1$ is the shear parameter (second parameter) of the soil $(\mathrm{N}), \rho$ is the mass per unit length of the beam $(\mathrm{kg} / \mathrm{m}), \mathrm{c}$ is the coefficient of viscous damping per unit length of the beam $(\mathrm{Ns} / \mathrm{m} 2), \mathrm{P}(\mathrm{x}, \mathrm{t})$ is the applied moving load per unit length $(\mathrm{N} / \mathrm{m}), \mathrm{x}$ is the space coordinate measured along the length of the beam (m), $t$ isthe time (s).

If loads are moving in the positive $\mathrm{x}$ direction with a constant advance velocity v, a moving coordinate $\xi$ can be defined by $(\mathrm{x}-\mathrm{vt})$. Then, the governing differential equation in a moving Cartesian coordinate system can be expressed as

$E I \frac{\partial^{4} w}{\partial x^{4}}-k_{1} \frac{\partial^{2} w}{d x^{2}}+k w+\rho \frac{\partial^{2} w}{\partial t^{2}}+c \frac{d w}{d t}=P \delta \quad x-v t \quad E q .2$

Where $\mathrm{P}$ is the concentrated load moves with a constant velocity $\mathrm{V}, \mathrm{P}(\mathrm{x}, \mathrm{t})=\mathrm{P} \delta(\mathrm{x}-\mathrm{vt})$ where $\delta$ is the Dirac's delta function and $\mathrm{x}$ is measured from the location of the load at $\mathrm{x}=$ 0 .

Using the two parameter model, the values of $\mathrm{k}$ and $\mathrm{k} 1$ are based on the constrained deformation of an elastic layer given by Vlazov and Leotiev [9]. For a single layer of thickness $\mathrm{H}$ with a linear variation of normal stresses, $\mathrm{k}$ and $\mathrm{k} 1$ per unit width are given by

$$
\bar{k}=\frac{E_{s}}{H 1+v_{s} 1-2 v_{s}} ; k_{1}=\frac{E_{s} H}{61+v_{s}}
$$

Divide both sides of Eq. (1) by EI to get

$$
\frac{\partial^{4} w}{d x^{4}}-\frac{k_{1}}{E I} \frac{\partial^{2} w}{\partial x^{2}}+\frac{k}{E I} w+\frac{\rho}{E I} \frac{\partial^{2} w}{\partial t^{2}}+\frac{c}{E I} \frac{\partial w}{\partial t}=\frac{P}{E I} \delta x-v t \quad E q .3
$$

Now define

$a=\frac{\rho}{2 E I} ; b^{2}=\frac{k}{E I} ; c_{1}=\frac{k_{1}}{2 E I} ; d=\frac{c}{E I}$

For an infinite beam in the steady state, it is found that the response $\mathrm{w}$ becomes a function of $(\mathrm{x}-\mathrm{vt})$, rather than of $(\mathrm{x}, \mathrm{t})$. The issue of the stability of the moving mass on beam becomes easier to solve if the reference frame is related to the moving mass. Thus, the Dirac delta function that locates the position of the contact point between the moving mass and the beam becomes a space-dependent function only.

Hence writing $\xi=x-v t$

The boundary conditions are as follows:

$w_{1} 0=w_{2} 0 ; w_{1}^{\prime} 0=w_{2}^{\prime} 0$

$w_{1}^{\prime \prime} 0=w_{2}^{\prime \prime} 0 ; w_{1}^{\prime \prime \prime} 0-w_{2}^{\prime \prime \prime} 0=\frac{P}{E I} \quad E q .4$

Infinite beam on a two parameter foundation model

For two parameter model foundation model considering damping the Eq. (3) can be written as follows.

$\frac{\partial^{4} w}{d x^{4}}-2 c_{1} \frac{\partial^{2} w}{\partial x^{2}}+b^{2} w+2 a \frac{\partial^{2} w}{\partial t^{2}}+d \frac{\partial w}{\partial t}=\frac{P}{E I} \delta x-v t \quad E q .5$
Reducing the above Eq. as

$$
\frac{\partial^{4} w}{d \xi^{4}}-2 c_{1} \frac{\partial^{2} w}{\partial \xi^{2}}+b^{2} w+2 a v^{2} \frac{\partial^{2} w}{\partial \xi^{2}}-d v \frac{\partial w}{\partial \xi}=0 \quad E q .6
$$

solution of the above Eq. is $w=e^{m \xi}$ and substituting in Eq. (6)

$m^{4}-2 c_{1} m^{2}+2 a v^{2} m^{2}+b^{2}-d v m=0$

Defining critical damping

as $d_{c r}=2 \sqrt{2} b \sqrt{a} ; d<d_{c r}$ constitutes the under damped case and critical velocity of the moving load $v_{c r}=\sqrt{\left(b+c_{1}\right) / a}$, the roots of the Eq. (7) are in the form

$$
\begin{aligned}
& m_{1}=-p+i q ; m_{2}=-p-i q, \\
& m_{3}=p+i r ; m_{4}=p-i r,
\end{aligned}
$$

where $\mathrm{p}, \mathrm{q}$ and $\mathrm{r}$ are real positive numbers.

Hence solution of the Eq. (7) can be written as

$$
\begin{aligned}
& w_{1} \xi=e^{-p \xi} A \cos q \xi+B \sin q \xi \text { for } \xi>0 \\
& w_{2} \xi=e^{p \xi} C \cos r \xi+D \sin r \xi \text { for } \xi<0 \quad E q .8 \\
& \text { Using Eq. (4) and (8) } \\
& A-C=0 \\
& -2 p A+B q-D r=0 \\
& r^{2}-q^{2} A-2 p q B-2 p r D=0 \\
& p\left(3 q^{2}-2 p^{2}+3 r^{2}\right) A+q 3 p^{2}-q^{2} B+r\left(r^{2}-3 p^{2}\right) D=\frac{P}{E I}
\end{aligned}
$$

The values of A, B, C and D have been solved analytically.

\section{PARAMETRIC STUDIES}

In this section, parametric study is conducted in order to get the response of moving loads in underdamed case. Default values of parameters used for numerical computation. These parameters reflect typical structural and material properties of pavement. Using the parametric value deflection has been calculated under the both cases using the above formulation. The variation in deflection and bending moment with new coordinate system $\xi$ are compared for both the cases.

Table 1 Soil and Beam Parameters

\begin{tabular}{|l|l|}
\hline Parameters & $\begin{array}{l}\text { Assumed } \\
\text { values }\end{array}$ \\
\hline$\rho(\mathrm{kg} / \mathrm{m})$ & 25 \\
\hline $\mathrm{EI}(\mathrm{Nm} 2)$ & $1.75 \times 106$ \\
\hline $\mathrm{k}(\mathrm{N} / \mathrm{m} 2)$ & $40.78 \times 105$ \\
\hline $\mathrm{k} 1(\mathrm{~N})$ & 666875 \\
\hline $\mathrm{P}(\mathrm{N} / \mathrm{m})$ & $93.36 \times 103$ \\
\hline $\mathrm{Es}(\mathrm{N} / \mathrm{m} 2)$ & $3.73 \times 106$ \\
\hline vs & 0.4 \\
\hline
\end{tabular}

\section{RESULTS AND DISCUSSION}

\subsection{Deflection Moment with Damping}

Because of the damping effect, the maximum dynamic displacement appears after the load passes through the point at $t=0$. Similar procedure is followed to get the response of the beam considering $10 \%$ damping ratio. The response has been found by using the formulation meant for damping in Eq. (8) 
considering four different critical velocity ratio i.e. $0.25,0.5$, 0.75 and 0.99 . From Fig.1, it is observed that the deflection is maximum at $\mathrm{x}=0$ at time $\mathrm{t}=0$. With increase of $\mathrm{x}$ the response of deflection gradually decreases. Thus due to damping effect the reaction of the moving load is delayed. From Figs. 1 to 4 , it can be concluded that with increase of velocity ratio maximum dynamic deflection increases. From Fig 4 it can be observed that at $\mathrm{x}=50 \mathrm{~m}$ the deflection is high as compared to the Fig.3. Here it may be inferred that load velocity has an impact such kind of response. All the steady state solutions are asymmetric with respect to the loading point. In particular, the maximum deformation into the foundation seems to lag behind the moving load.

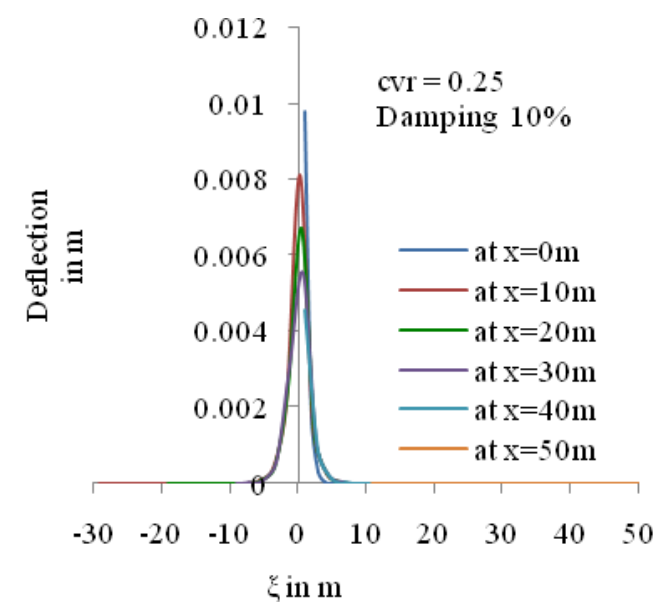

Fig.1 Deflection Vs Distance with velocity ratio $=0.25$

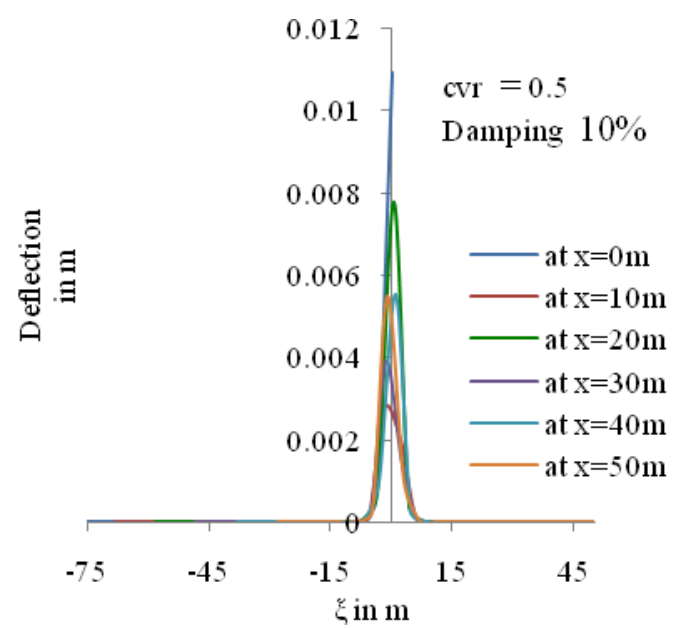

Fig.2 Deflection Vs Distance with velocity ratio $=0.5$

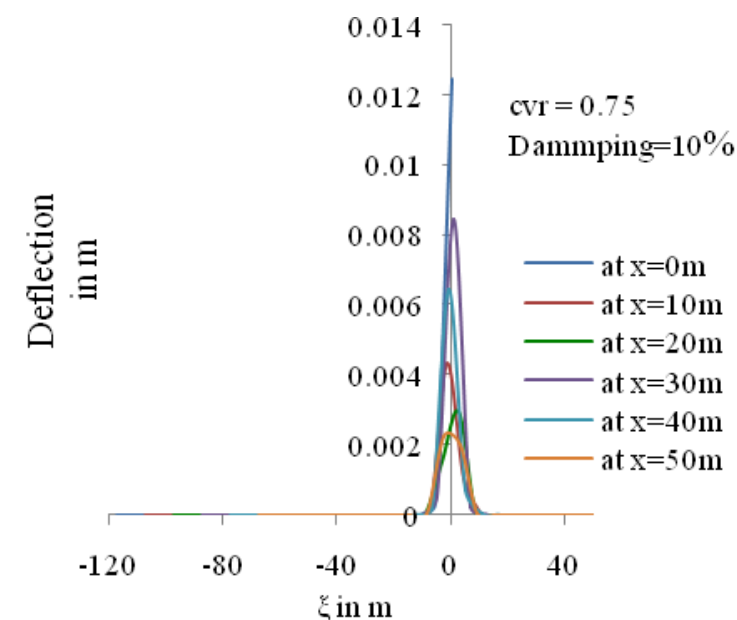

Fig.3 Deflection $V s$ Distance with velocity ratio $=0.75$

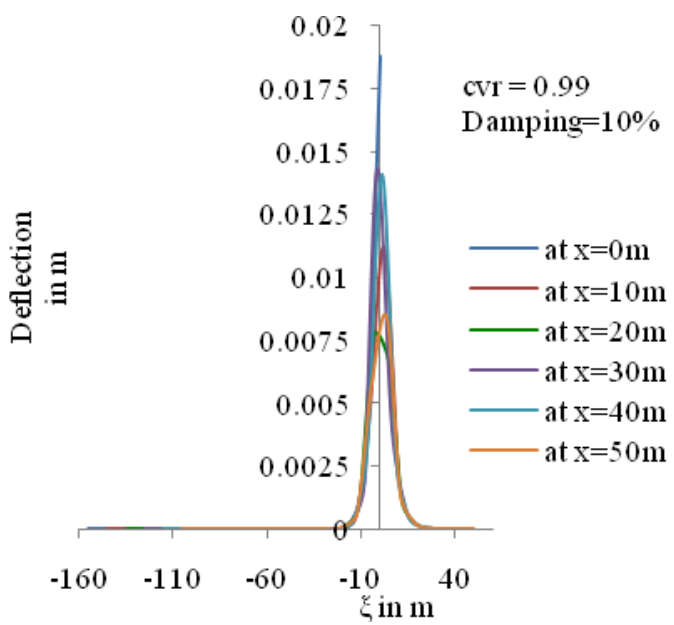

Fig.4 Deflection Vs Distance with velocity ratio $=0.99$

\subsection{Bending Moment with Damping}

Due to inclusion of $10 \%$ damping there is a dissipation of energy along the railway track. From Figs.15 to 16, it is observed that the effect of negative bending moment has reduced as compared to the positive bending moment hence there is uplift behind the moving load. Hence there will be less settlement of the track due to presence of the damping. From comparison of Figs.15 to 18, it is observed that with increase of critical velocity ratio higher value of uplift is found from bending moment profile. However due to higher critical velocity in Fig. 18 there is considerable amount of deformation found due to negative bending moment. Also with increasing distance away from the loading point the bending profile get diminished. 


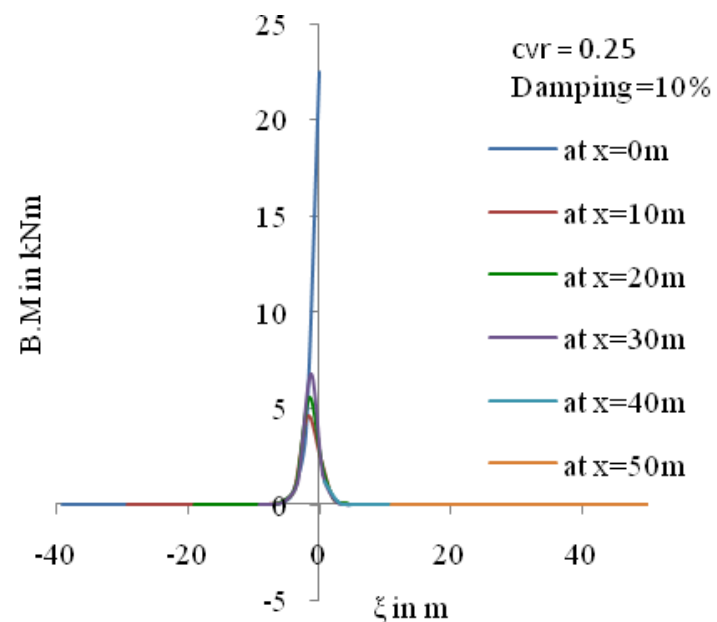

Fig.5 B.M Vs Distance with velocity ratio $=0.25$

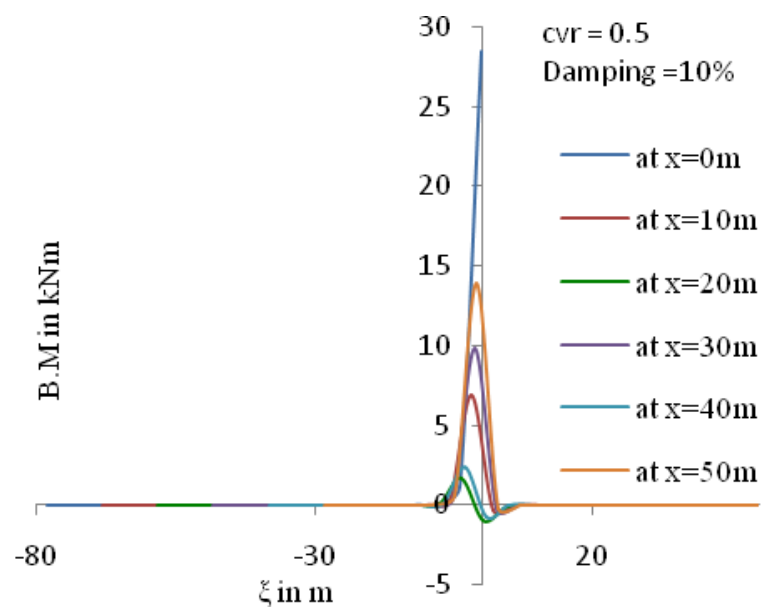

Fig.6 B.M Vs Distance with velocity ratio $=0.5$

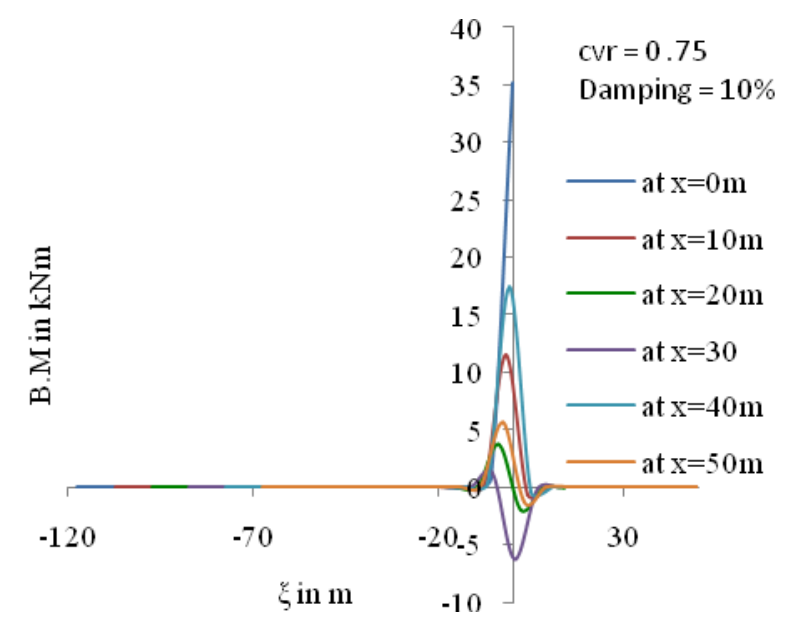

Fig.7 B.M Vs Distance with velocity ratio $=0.75$

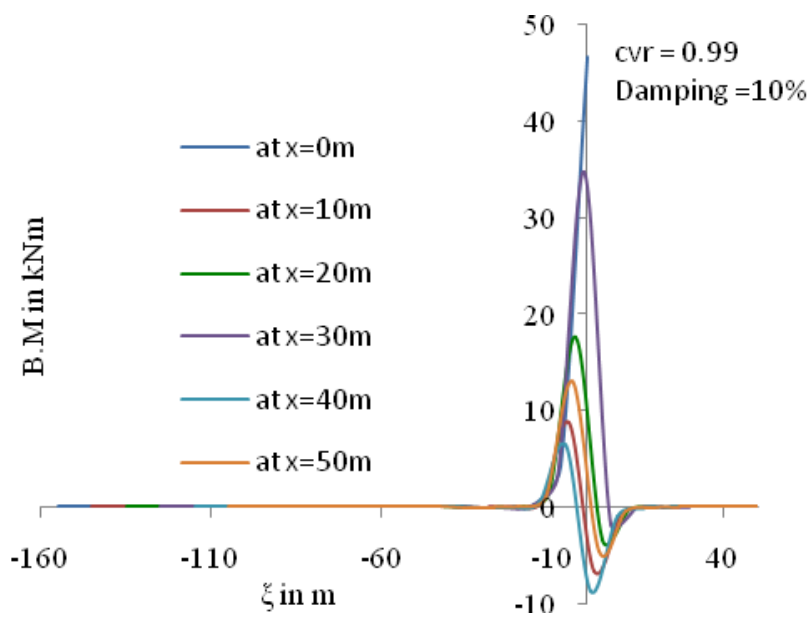

Fig.8 B.M Vs Distance with velocity ratio $=0.99$

\section{CONCLUSION}

A Bernoulli-Euler beam resting on an elastic foundation subject to moving dynamic loads is studied in this paper. The steady-state deflection has been studied and compared under damping. The deflection response of the beam with elastic foundation is symmetric with respect to time $t=0$ or space $\mathrm{x}=0$, while it is asymmetric when damping is considered. The maximum displacement occurs behind the moving load. The configurations of the steady state solutions may change as damping varies. At subcritical speed the absolute value of the deflection of the beam increases with increase of the load velocity. Peak maximum deflection appears when the load travels at the critical speed.

\section{REFERENCES}

[1] Cifuentes, A.O., (1989). Dynamic response of a beam excited by a moving mass. FiniteElements in Analysis and Design.237-246.

[2] Harr, M. E., Davidson J. L. and Ramaswamy, S. V. (1969). Euler Beams on a two parameter foundation model. ASCE .J. Soil.Mechanics and Foundation. 95, 933-948

[3] Fryba, L. (1972). Vibration of solids and structures under moving loads. Noordhoff International Publishing, Groningen, The Netherlands

[4] J.T. Kenny Jr., Steady state vibrations of beam on elastic foundation for moving load, Journal of Applied Mechanics, Transactions ASME 21 (1954) 359-364..

[5] Kien, N. D. (2008). Dynamic response of prestressedtimoshenko beams resting on two-parameter foundation to moving harmonic load. TechnischeMechanik. 28(3-4),237-258.

[6] Mallik, A. K., Chandra, S., \& Singh, A. B. (2006).

Steady-state response of an elastically supported infinite beam 
International Journal of Science and Engineering Applications (IJSEA)

Volume 1 Issue 2, 2012, ISSN - 2319-7560 (online)

to a moving load. Journal of Sound and Vibration, 291(3-5), 1148-1169.

[7] Sun, L.,Feiquan,Luo (2008). Steady-State Dynamic Response of a Bernoulli-Euler Beam on a Viscoelastic Foundation Subject to a Platoon of Moving Dynamic Loads. J. Appl. Mech. ASME. 130,051002-3-19.

[8] Zhaohua, F. and Cook, R. D. (1983). Beam elements on two-parameter elastic foundations. J. Eng. Mech .Div., ASCE.
109, 1390-1402. F. Richards, S. E. Davidson and S.A Long, Dual bandreactively loaded microstrip patch antenna, IEE Trans. Antenna Propa., 33 (1985) 556-561.

[9] Vlasov, V. Z. and U. N. Leontiev (1966) Beams, Plates, and Shells on Elastic Foundation.Israel Program for Scientific Translations, Jerusalem. (translated from Russian) 